\title{
GMR
}

\section{Combined effect of traditional Chinese and Western medicine on inflammatory factors in patients with diabetes-induced xerophthalmia}

\author{
S.Q. Wu, Q. Dai, J.L. Xu, W.Y. Sheng, Q.B. Xu and L.Y. Zhong \\ Department of Ophthalmology, Hangzhou Red Cross Hospital, Hangzhou, \\ Zhejiang, China \\ Corresponding author: Q. Dai \\ E-mail: qidaiasd@sina.com
}

Genet. Mol. Res. 15 (4): gmr15049030

Received July 26, 2016

Accepted September 14, 2016

Published November 3, 2016

DOI http://dx.doi.org/10.4238/gmr15049030

Copyright $(\underset{0}{ } 2016$ The Authors. This is an open-access article distributed under the terms of the Creative Commons Attribution ShareAlike (CC BY-SA) 4.0 License.

\begin{abstract}
Diabetes-induced xerophthalmia is a general metabolic disorder with high incidence and increased treatment difficulty. Our study aimed to explore the combined effect of traditional Chinese and Western medicines on diabetes-associated xerophthalmia. We recruited 60 diabetic xerophthalmia patients, and randomly assigned them to either the control (Western medicine treatment) or the experimental (combined treatment of traditional Chinese medicine and Western medicine) groups. Pre-treatment and post-treatment analyses were performed to assess the combined therapeutic effect of traditional Chinese and Western medicine on xerophthalmia-associated indicators. We found that the experimental group expressed reduced levels of IL-1, IL-8, and TNF- $\alpha(\mathrm{P}<0.05)$ as compared to the control group. Furthermore, the experimental group showed higher treatment efficacy as compared to the control group ( 85.00 vs $51.67 \% \mathrm{Z}=22.244, \mathrm{P}$ $<0.05)$. In addition, break-up time $(\mathrm{t}=20.582, \mathrm{P}<0.05)$ and tear
\end{abstract}


section $(\mathrm{t}=23.082, \mathrm{P}<0.05)$ was increased in the experimental group as compared to the controls. Lastly, it was found that the combined treatment of traditional Chinese and Western medicine effectively reduced corneal injuries, as indicated by reduced fluorescein staining. This study suggested that a combination treatment consisting of both traditional Chinese and Western medicines may be effective against xerophthalmia in diabetes, and that inflammatory factors are potential biomarkers to examine the treatment efficacy.

Key words: Combined treatment; Traditional Chinese medicine; Diabetes; Xerophthalmia; Tear; Inflammatory factors

\section{INTRODUCTION}

Xerophthalmia refers to the pathological condition in which the eye fails to produce tears, resulting in dryness of the tear film and subsequent ocular symptoms (Wladis, 2012). Previous clinical studies have demonstrated that diabetes is one of the leading causes of xerophthalmia. In addition, diabetes-induced xerophthalmia can lead to cataracts, glaucoma, as well as retinal degeneration, and is one of the main causes of blindness in clinical settings (Lee et al., 2012; Murakami and Manche, 2012; Yaguchi et al., 2012). Moreover, most patients with diabetes suffer from dryness of the eye, foreign body sensations, photophobia, and vision fluctuations (Clark and Eggenberger, 2012). Recent studies suggested that aside from apoptosis and neuroregulation, inflammation is another important factor in the pathogenesis of xerophthalmia (Enríquez-de-Salamanca et al., 2010; Swymer and Neville, 2012; Ghosh et al., 2014). Diabetic xerophthalmia is a complicated disease with complex mechanisms, and is characterized as follows: 1) elevated blood glucose in tears, resulting in increased osmotic pressure and changes in tear components. 2) Decreased mucus secretion due to depleted numbers of goblet cells, which in turn reduces lubrication of the saccus conjunctivae, and increases friction between the eyelid and the eyeball. 3) Increased advanced glycation end products, which damage lacrimal epithelial cells, and leads to reduced tear secretion. 4) Altered distribution, morphology, and structure of the cornea nerve, which causes decreased corneal sensitivity, blocked tear secretion, reduced blinking, accelerated tear evaporation, and shortened break-up time (BUT) (Kamoi et al., 2012; Fuerst et al., 2014; Tan et al., 2014; Vanathi et al., 2014). We hypothesized that ocular inflammation may be detected in the tear components of diabetic xerophthalmia patients, and that treatment efficacy could be assessed via changes in inflammatory factors in tears.

Previous studies showed that traditional Chinese medicine plays an important role in the evaluation and treatment of several diseases, such as diabetes (Covington, 2001) and vascular cognitive impairments (Wang et al., 2015). A report on integrated Chinese and Western treatments of diabetes was published in 1987 (Yao, 1987). A past clinical trial showed that ten patients who responded poorly to Western drugs also failed to respond to Chinese herbs (Gao, 1989). Interestingly, significant improvements were observed in eight of the ten patients after they were given both the herbs and drugs concurrently, suggesting that combined treatments of traditional Chinese and Western medicine could alleviate symptoms of diabetes. However, whether this combination therapy could improve the treatment efficacy of diabetes-associated xerophthalmia remains poorly understood. Therefore, our study aimed to investigate the

Genetics and Molecular Research 15 (4): gmr15049030 
efficacy of Chinese/Western combination treatment in diabetic xerophthalmia by examining the secretion of multiple inflammatory factors, including IL- $1 \alpha$, IL-1 $\beta$, IL-6, IL-8, and TNF- $\alpha$.

\section{MATERIAL AND METHODS}

\section{Clinical samples}

Patients (60) with diabetes-induced xerophthalmia were recruited from the Hangzhou Red Cross Hospital between January 1, 2015 and December 31, 2015. Subjects were randomly assigned to either the control (Western medicine) or the experimental group (combination of traditional Chinese and Western medicine).

The study protocol has been approved by the Ethics Committee of Hangzhou Red Cross Hospital. Written informed consents were obtained from all subjects prior to enrollment.

\section{Screening criteria}

The inclusion criteria were as follows: 1) patients with confirmed diabetes-induced xerophthalmia; 2) between 17 and 76 years of age; 3) informed consent has been given.

The exclusion criteria were as follows: 1) pregnancy; 2) history of diseases that affect vital organs, including heart, liver, and kidney; 3) mental disorder; 4) need for long-term eye drops therapy.

\section{Therapeutic method}

All patients were treated for three months. Therapeutic methods were as follows: 1) control group: Western medicine treatment. Artificial tears without preservatives were used six times a day. 2) Experimental group: combined treatment of traditional Chinese and Western medicine. The treatment principle was to strengthen the body's resistance to pathogenic factors, through dispelling the pathogenic wind and nourishing Yin. According to the different diabetes subtypes, three traditional Chinese treatments were implemented, including Qiwei Baizhu powder for deficiencies in both Qi and Yin, Zhibai Dihuang decoction for deficiencies in Yin and interior heat, as well as a combination of Liuwei Dihuang decoction and kidney-Qi pills for Yin-Yang deficiency.

\section{Observation targets}

\section{Symptom scoring}

Patients were required to fill out a questionnaire. Scores were given on dryness of the eye, fatigue, itching of the eye, presence of foreign bodies, burning sensations, viscous secretion, fear of wind, photophobia, and sensitivity to stimulus.

\section{Ocular surface scoring}

Scoring was performed as previously described (Jeroudi and Yeh, 2014). The OCULUS Keratograph ocular surface analyzer was used to assess the BUT.

Genetics and Molecular Research 15 (4): gmr15049030 


\section{Fluorescein staining}

The cornea was divided into four quadrants. All quadrants were sequentially scored. A score of 0 represented no staining; a score of 1 represented 1-30 staining dots; a score of 2 scores represented $>30$ staining dots; a score of 3 scores represented total staining with ulcer and filaments.

\section{Schimer I test}

Basal tear secretion was assessed via the Schimer I test, as previously described (Jeroudi and Yeh, 2014).

\section{Inflammation factors in tears}

Test papers with tear samples were stored in $1-\mathrm{mL}$ tubes at $-20^{\circ} \mathrm{C}$. Extracting solution $(0.5$ $\mathrm{M} \mathrm{NaCl}, 0.5 \%$ Tween 20$)(200 \mu \mathrm{L})$ was added to the tubes at room temperature, and were allowed to incubate for $3 \mathrm{~h}$. Test papers were then removed, the remaining extracting solution was pipetted into 2-mL tubes, and centrifuged ( $1000 \mathrm{rpm}$ ) for $10 \mathrm{~s}$. Levels of inflammation factors were assessed with Human Cytokine 16-Plex ELISA Kit (PBL Assay Science, Piscataway, NJ, USA)

\section{Compliance}

Patient compliance was determined as previously described (Noh and Kim, 2013). Briefly, compliance of each group was calculated as the number of patients receiving three months treatment / (total number of patients - number of patients without full data) x $100 \%$.

\section{Side effects}

Side effects were evaluated as previously described (Mostafa et al., 2012). Incidence rate of side effects were calculated as follows: number of exclusive patients due to side effects / (total number of patients - number of patients without full data) x $100 \%$.

\section{Statistical analysis}

The SPSS 19.0 software was used for data processing. Measurement data are reported as means \pm standard deviation. The paired Student $t$-test was performed to compare between pre- and post-treatment data within the same population. The unpaired Student $t$-test was used to compare the various parameters between control and experimental groups. Chi-squared test was used to analyze the effective rate of the treatment. $\mathrm{P}<0.05$ was considered statistically significant.

\section{RESULTS}

\section{Examination of five inflammation factors}

As shown in Table 1, both control and experimental groups showed significant changes in the expression levels of multiple inflammatory factors $(\mathrm{P}<0.05)$, including IL-1 $\alpha$, IL-6, IL8 , and TNF- $\alpha$. As compared with the control group, the experimental group exhibited higher treatment efficacy rate $(51.67<85.00 \%, \mathrm{Z}=22.244, \mathrm{P}<0.05)$. 


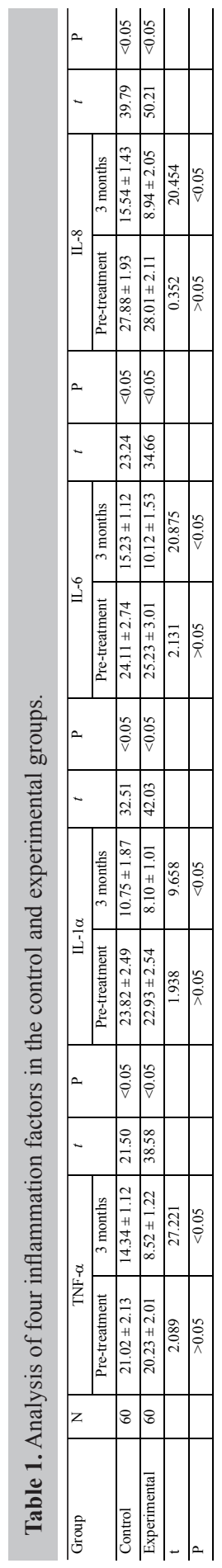

Genetics and Molecular Research 15 (4): gmr15049030 


\section{Combined treatment alleviated clinical symptoms and increased therapeutic effective rate}

Based on the questionnaires, it was found that multiple parameters were improved following treatments, with the experimental group showing greater improvements as compared to the control group (Figure 1). Moreover, combined treatment showed higher therapeutic effective rate as compared to Western medicine alone $(51.67<85.00 \%, \mathrm{Z}=22.244, \mathrm{P}<0.05$, Table 2$)$.

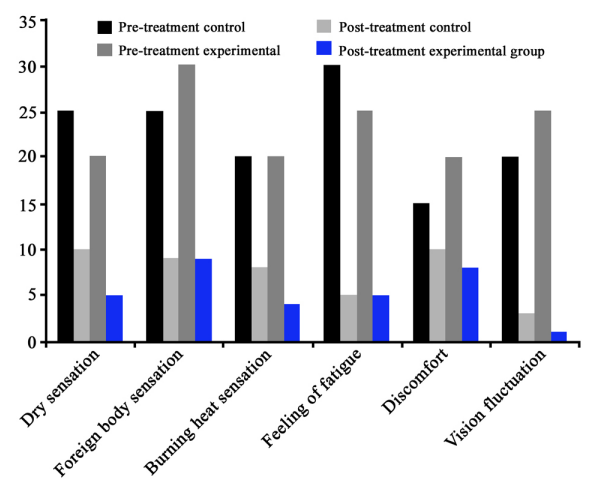

Figure 1. Histogram of clinical symptoms under various treatment conditions.

Table 2. Effective rate of treatments in control and experimental groups.

\begin{tabular}{l|c|c|c|c|c}
\hline Group & Eyes & Cured & Improved & Inefficient & Effective rate (\%) \\
\hline Control & 60 & $4(6.67)$ & $27(45.00)$ & $29(48.33)$ & 51.67 \\
\hline Experimental & 60 & $21(35.00)$ & $30(50.00)$ & $9(15.00)$ & \\
\hline$Z$ & & 22.244 & & & 85.00 \\
\hline$P$ & & $<0.05$ & & & \\
\hline
\end{tabular}

\section{Combined treatment improved prognosis of diabetic xerophthalmia}

As compared with the control group, the experimental group demonstrated better examination results, including BUT, the Schirmer I test, and positive fluorescein staining (Table 3).

Table 3. Analysis of multiple parameters in control and experimental groups.

\begin{tabular}{l|c|c|c|c}
\hline Group & Eyes & BUT $($ mean \pm SD; $\mathrm{s})$ & Schirmer I (mean \pm SD, mm/5 min) & FLS (\%) \\
\hline Control & 60 & $11.55 \pm 2.51$ & $11.10 \pm 2.98$ & $6.67(2 / 30)$ \\
\hline Experimental & 60 & $8.09 \pm 2.68$ & $7.19 \pm 3.11$ & $30.00(9 / 30)$ \\
\hline$t$ & & 7.299 & 7.032 & 10.132 \\
\hline $\mathrm{P}$ & & $<0.05$ & $<0.05$ & $<0.05$ \\
\hline
\end{tabular}

\section{Combined treatment increased BUT and tear secretion}

As shown in Table 4, the experimental group not only exhibited prolonged BUT ( $t=$ 20.582, $\mathrm{P}<0.05)$, but also higher tear secretion $(t=23.082, \mathrm{P}<0.05)$, as compared with the control group.

Genetics and Molecular Research 15 (4): gmr15049030 
Table 4. Analysis of BUT and tear secretion in control and experimental groups.

\begin{tabular}{l|c|c}
\hline Group & Increment of BUT $(\Delta \mathrm{s})$ & Increment of tear secretion $(\Delta \mathrm{mm} / 5 \mathrm{~min})$ \\
\hline Control & $2.33 \pm 0.92$ & $3.39 \pm 1.05$ \\
\hline Experimental & $6.12 \pm 1.09$ & $8.51 \pm 1.36$ \\
\hline$t$ & 20.582 & 23.082 \\
\hline $\mathrm{P}$ & $<0.05$ & $<0.05$ \\
\hline
\end{tabular}

\section{DISCUSSION}

As a whole, ocular surface tissues serve as a single functional unit due to their closed anatomical and functional relationship. These structures regulate tear secretion and formation of the tear film to maintain the integrity and normal function of the tear film. Abnormality in any part of ocular surface tissues could result in xerophthalmia (Malik et al., 2013; Shamsheer and Arunachalam, 2015). Diabetic patients often show incomplete conjunctival epithelium, which leads to difficulties in tear film attachment and a reduction in the numbers of goblet cells. This leads to reduced lubrication from the saccus conjunctivae, increased friction between the eyelid and the eyeball, and increased vulnerability of the tear film (Liu and Li, 2014; DeMill et al., 2015). In addition, patients with diabetic xerophthalmia may have decreased corneal sensation, blocked tear reflection secretion, reduced numbers of blinks, accelerated tear evaporation, and shortened BUT. All above changes can further exacerbate symptoms of xerophthalmia (Liu et al., 2015; Najafi et al., 2015).

Our study explored the efficacy of a combination treatment using both traditional Chinese and Western medicine on diabetes-induced xerophthalmia. In addition, different traditional Chinese medicines were applied to the three subtypes of diabetic xerophthalmia, which were deficiencies in both Qi and Yin, deficiencies in Yin and interior heat, and Yin-Yang deficiency. We found that the four inflammation factors were significantly decreased following administration of the combination treatment, suggesting that this therapy may be more effective against inflammation than Western medicine alone. Furthermore, patients treated with the combination therapy demonstrated better prognosis as compared to the controls, as shown by changes in BUT, the Schirmer I test, and fluorescein staining. Combined treatment also alleviated symptoms of diabetic xerophthalmia, and improved functions of the tear film. This study also demonstrated that inflammation factors could be used as potential biomarkers to assess therapeutic efficacy.

In conclusion, our study demonstrated that diabetic xerophthalmia was associated with inflammation, and that combined treatment of traditional Chinese and Western medicine is a promising therapy to attenuate inflammation. Traditional Chinese medicine may be able to alleviate the symptoms and improve prognosis for patients with diabetes xerophthalmia.

\section{Conflicts of interest}

The authors declare no conflict of interest.

\section{ACKNOWLEDGMENTS}

Research supported by the Traditional Chinese Medicine Science and Technology Project of Zhejiang Province (\#2016ZA151). 


\section{REFERENCES}

Clark D and Eggenberger E (2012). Neuro-ophthalmology of movement disorders. Curr. Opin. Ophthalmol. 23: 491-496. http://dx.doi.org/10.1097/ICU.0b013e328358ba14

Covington MB (2001). Traditional Chinese medicine in the treatment of diabetes. Diabetes Spectr. 14: 154-159. http:// dx.doi.org/10.2337/diaspect.14.3.154

DeMill DL, Hussain M, Pop-Busui R and Shtein RM (2015). Ocular surface disease in patients with diabetic peripheral neuropathy. Br. J. Ophthalmol. 100: 924-928.

Enríquez-de-Salamanca A, Castellanos E, Stern ME, Fernández I, et al. (2010). Tear cytokine and chemokine analysis and clinical correlations in evaporative-type dry eye disease. Mol. Vis. 16: 862-873.

Fuerst N, Langelier N, Massaro-Giordano M, Pistilli M, et al. (2014). Tear osmolarity and dry eye symptoms in diabetics. Clin. Ophthalmol. 8: 507-515.

Gao JZ (1989). Treatment of diabetes with Shen Qi Tao Hong Tang. J. Zhejiang Tradit. Chin. Med. Coll. 13: 15-16.

Ghosh S, Ghosh S, Azharuddin M, Bera S, et al. (2014). Change in tear protein profile in diabetic retinopathy with duration of diabetes. Diabetes Metab. Syndr. 8: 233-235.http://dx.doi.org/10.1016/j.dsx.2014.09.019

Jeroudi A and Yeh S (2014). Diagnostic vitrectomy for infectious uveitis. Int. Ophthalmol. Clin. 54: 173-197. http:// dx.doi.org/10.1097/IIO.0000000000000017

Kamoi M, Ogawa Y, Nakamura S, Dogru M, et al. (2012). Accumulation of secretory vesicles in the lacrimal gland epithelia is related to non-Sjögren's type dry eye in visual display terminal users. PLoS One 7: e43688. http://dx.doi. org/10.1371/journal.pone.0043688

Lee SY, Petznick A and Tong L (2012). Associations of systemic diseases, smoking and contact lens wear with severity of dry eye. Ophthalmic Physiol. Opt. 32: 518-526.http://dx.doi.org/10.1111/j.1475-1313.2012.00931.x

Liu H, Sheng M, Liu Y, Wang P, et al. (2015). Expression of SIRT1 and oxidative stress in diabetic dry eye. Int. J. Clin. Exp. Pathol. 8: 7644-7653.

Liu ZY and Li CR (2014). Treatment of dry eye caused by meibomian lang dysfunction. Int Eye Sci 14: 270-272.

Malik K, Matejtschuk P, Thelwell C and Burns CJ (2013). Differential scanning fluorimetry: rapid screening of formulations that promote the stability of reference preparations. J. Pharm. Biomed. Anal. 77: 163-166. http://dx.doi. org/10.1016/j.jpba.2013.01.006

Mostafa S, Seamon V and Azzarolo AM (2012). Influence of sex hormones and genetic predisposition in Sjögren's syndrome: a new clue to the immunopathogenesis of dry eye disease. Exp. Eye Res. 96: 88-97. http://dx.doi. org/10.1016/j.exer.2011.12.016

Murakami Y and Manche EE (2012). Prospective, randomized comparison of self-reported postoperative dry eye and visual fluctuation in LASIK and photorefractive keratectomy. Ophthalmology 119: 2220-2224. http://dx.doi. org/10.1016/j.ophtha.2012.06.013

Najafi L, Malek M, Valojerdi AE, Khamseh ME, et al. (2015). Dry eye disease in type 2 diabetes mellitus; comparison of the tear osmolarity test with other common diagnostic tests: a diagnostic accuracy study using STARD standard. $J$. Diabetes Metab. Disord. 14: 39. http://dx.doi.org/10.1186/s40200-015-0157-y

Noh JY and Kim WJ (2013). Influenza vaccines: unmet needs and recent developments. Infect. Chemother. 45: 375-386. http://dx.doi.org/10.3947/ic.2013.45.4.375

Shamsheer RP and Arunachalam C (2015). A clinical study of Meibomian gland dysfunction in patients with diabetes. Middle East Afr. J. Ophthalmol. 22: 462-466. http://dx.doi.org/10.4103/0974-9233.167827

Swymer C and Neville MW (2012). Tafluprost: the first preservative-free prostaglandin to treat open-angle glaucoma and ocular hypertension. Ann. Pharmacother. 46: 1506-1510.http://dx.doi.org/10.1345/aph.1R229

Tan X, Sun S, Liu Y, Zhu T, et al. (2014). Analysis of Th17-associated cytokines in tears of patients with dry eye syndrome. Eye (Lond.) 28: 608-613. http://dx.doi.org/10.1038/eye.2014.38

Vanathi M, Kashyap S, Khan R, Seth T, et al. (2014). Ocular surface evaluation in allogenic hematopoietic stem cell transplantation patients. Eur. J. Ophthalmol. 24: 655-666. http://dx.doi.org/10.5301/ejo.5000451

Wang LP, Zhang XY, Liu N, Ma ZZ, et al. (2015). Comparison of integrated traditional Chinese and western medicine therapy on vascular cognitive impairment with no dementia. Genet. Mol. Res. 14: 4896-4902. http://dx.doi. org/10.4238/2015.May.11.22

Wladis EJ (2012). Intraductal meibomian gland probing in the management of ocular rosacea. Ophthal. Plast. Reconstr. Surg. 28: 416-418. http://dx.doi.org/10.1097/IOP.0b013e3182627ebc

Yaguchi S, Ogawa Y, Kamoi M, Uchino M, et al. (2012). Surgical management of lacrimal punctal cauterization in chronic GVHD-related dry eye with recurrent punctal plug extrusion. Bone Marrow Transplant. 47: 1465-1469. http://dx.doi.org/10.1038/bmt.2012.50

Yao QG (1987). Integrated Chinese and Western treatment of 53 cases of diabetes. Chin. J. Integr. Med. 7: 363-364.

Genetics and Molecular Research 15 (4): gmr15049030 